\title{
Optical Driving for a Computer System with Augmented Reality Features
}

\author{
Tomasz Pałys \\ Military University of \\ Technology \\ Kaliskiego Str. 2, \\ 01-489 Warsaw, Poland, \\ Email: \\ tomasz.palys@wat.edu.pl
}

\author{
Krzysztof Murawski \\ Military University of \\ Technology \\ Kaliskiego Str. 2, \\ 01-489 Warsaw, Poland, \\ IEEE Member \# 92707852 \\ Email:
}

krzysztof.murawski@wat.edu.pl

\author{
Artur Arciuch \\ Military University of \\ Technology \\ Kaliskiego Str. 2, \\ 01-489 Warsaw, Poland, \\ Email: \\ artur.arciuch@wat.edu.pl
}

\author{
Andrzej Walczak \\ Military University of \\ Technology \\ Kaliskiego Str. 2, \\ 01-489 Warsaw, Poland, \\ Email: \\ andrzej.walczak@wat.edu.pl
}

\begin{abstract}
This article proposes a laser beam encoding method that is used to control an augmented reality system. Experiments were performed using a red laser emitting a wavelength of $\lambda=650 \mathrm{~nm}$ and a power of $P=3 \mathrm{~mW}$. The purpose of the study was to investigate the methods of modulation and demodulation of the encoded laser signal, and to examine the influence of parameters such as laser pulse duration, camera image resolution, the number of recorded frames per second on the demodulation result of the optical signal.

The results show that the proposed coding method provides the transmission of the necessary information in a single laser beam (no less than 36 codes with a decoding efficiency of 99.9\%). The developed coding method enables, based on the sequence analysis of video images, the influence on the course of the simulation performed in augmented reality, including distinguishing players and actions taken by them. This is an important advancement in relation to interaction systems used to influence augmented reality.
\end{abstract}

\section{INTRODUCTION}

$\mathrm{T}$ he impact on augmented reality $(A R)$ can occur while using different types of devices $[1-3]$. It consists in producing intentional and previously planned possible behavior of the system, which for the "player" will create the impression of interaction [4]. The coding and decoding system [5] plays a key role in such communication. Its most important parameters include the code capacity and the time of encoding and decoding the transmitted information. In the case under consideration, the data to be encoded is obtained from the user. They arise as a result of interacting with him/her through patterns or objects presented in the image that force the user to behave in a certain way. This behavior is recognized by it and analyzes the $A R$ system. For this purpose, markers [6, 7], 9 DOF sensors [8] or camera systems including 3D cameras $[9,10]$ are used. In the augmented reality system that is based on tags, the player is "stuck" with markers and then observed by an optical system consisting of multiple cameras. A similar effect is obtained when a player uses 9 DOF sensors to visualize its movement, Fig. 1. The position and activity of the player is then determined on the basis of data received from

This work was not supported by any organization accelerometers, gyroscopes and magnetometers [11]. In the solution shown in Fig. 1, 14 sensors were used to determine the position and movement of the player. In other designs, motion is determined using methods that determine local depth maps [12]. On their basis information undergoes synthesization and selected behaviors of the player are identified [13].

The commercial $A R$ system used in the study has been enhanced with a proprietary control system, the main components of which are the video camera (receiver), the signal decoder (microprocessor device) and the manipulator (mock-up weapon) along with the encoder (encoder). The transmitter uses amplitude modulation of the laser beam. The laser beam is reflected from the screen and recorded by the video camera. The attractiveness of the suggested approach is the ability to influence $A R$ even when the distance between the screen and the player reaches $600 \mathrm{~m}$.

The developed coding method eliminates problems identified as essential in [14]. The coding presented in paper [14] was based on the data transmission protocol used in the RS-232 standard.

\section{HARDWARE DESCRIPTION}

The laser beam modulation is implemented by the encoder, Fig. 2. The main component of the encoder is the AT89s8253 microcontroller clocked at $f=22.1184 \mathrm{MHz}$. The encoder performs the signal coding algorithm discussed in point IV. The result of the algorithm operation, depicted in the form of a sequence of logic signals (binary values), is
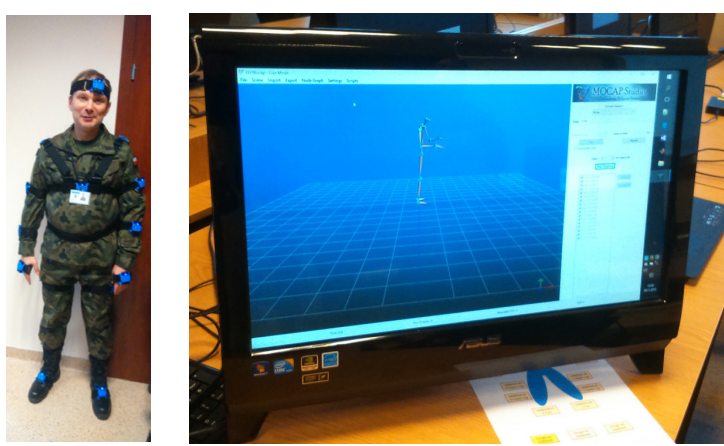

Fig. 1 View of player equipped with a 9 DOF sensors system 


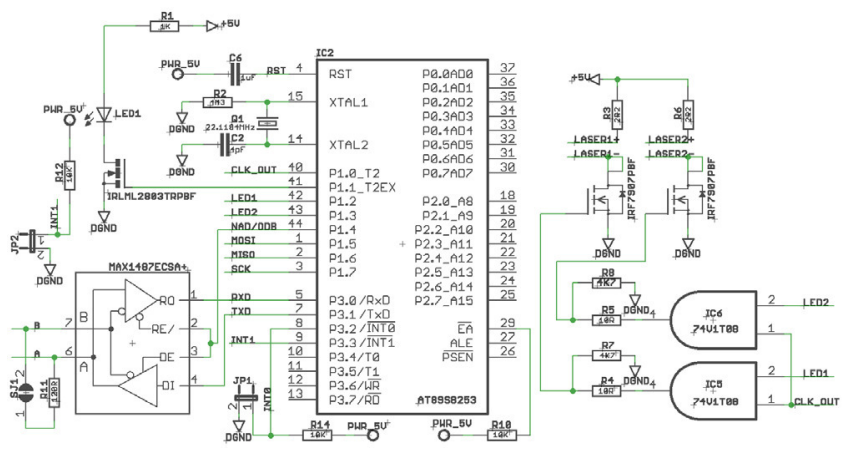

Fig. 2 Diagram of the laser beam modulation system (main elements)

taken out in series to the microcontroller terminals (indicated during configuration) - P1.2 or P1.3. These terminals, in the case of a "1" logic input, add power to the laser diodes power supply (not shown in Fig. 2). The developed version of the system and software makes it possible to simultaneously control two laser beams. In addition, they can be keyed by a binary signal or a rectangular wave generated by the microcontroller on the P1.0 terminal. This property was used in work [15 - 18] to control the brightness of the near-infrared illuminator. Activation of $\mathrm{P} 1.2$ or $\mathrm{P} 1.3$ terminals occurs after identifying the falling edge of the signal at the P3.2 terminal of the microcontroller (JP1 connector). In the presented layout, the button attached to the JP1 connector was taken out for the user. Similarly, in order to enable manual and remote configuration of the system, a button attached to the JP2 connector was taken out as well as communication connectors operating in the RS-485 standard. Giving a high signal on line P0.4 (as a result of JP2 connectors short-circuiting) puts the system into the configuration state. The button attached to the JP1 connector is then used to select the player identifier (Player ID) in the computer system. The selection is signaled to the user by a LED attached to the P1.1 terminal of the microcontroller and stored in a non-volatile memory (EEPROM). These actions can also be performed from the parent program when an encoder device is attached to a PC computer using a communications connector.

a)

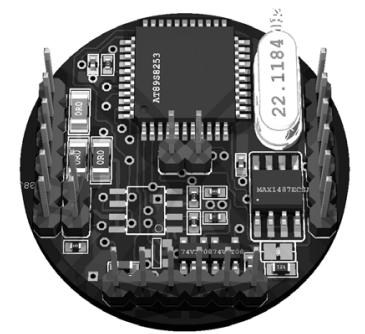

b)

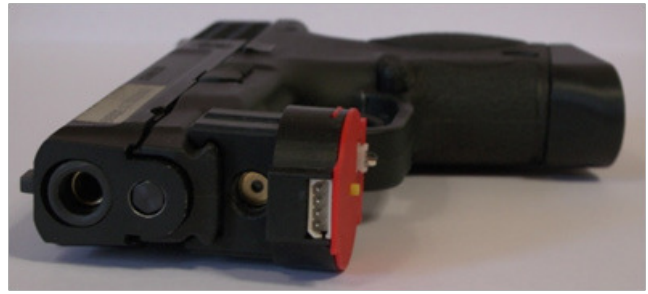

Fig. 3 Laser signal encoding module (a), view of installed device (b) a)

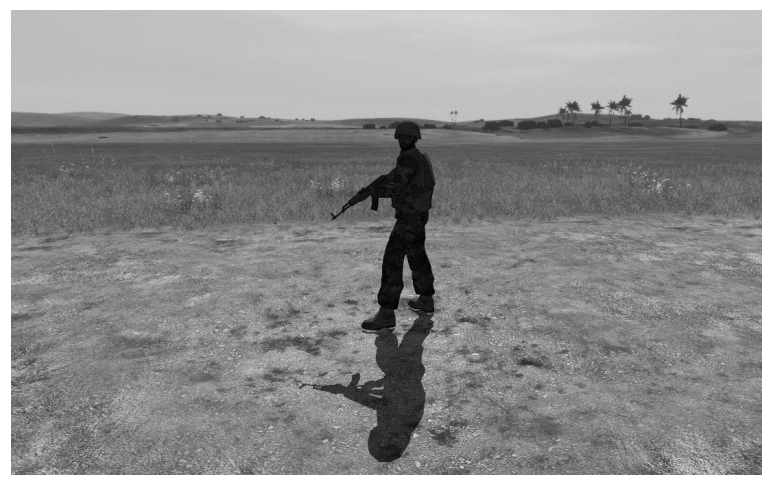

b)

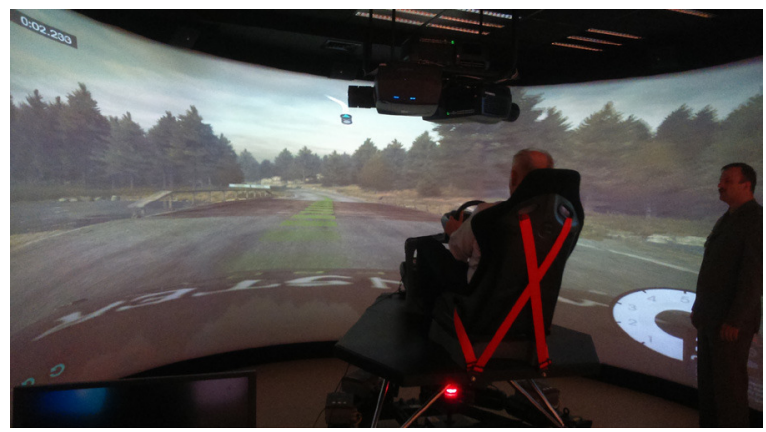

Fig. 4 Augmented reality environment: fram example (a), visualization system along with the screen in the shape of a half cylinder (b)

The view of the laser coding system is shown in Fig. 3a, and its location on a model of a gun is shown in Fig. $3 \mathrm{~b}$.

\section{MEASUREMENT SYSTEM CONFIGURATION}

The study of laser beam coding and decoding techniques was performed under controlled laboratory conditions. The experiment was conducted using the Manta G-201 Allied Vision camera equipped with a focal length lens of $F=8 \mathrm{~mm}$ and a bandpass filter for which $\lambda$ was equal $650 \mathrm{~nm}$ and the window width $\Delta \lambda$ was $\pm 10 \mathrm{~nm}$, and the OptiTrack 120 Slim camera with a lens with a focal length of $F=8 \mathrm{~mm}$. The laser beam produced by a laser module with a power of $\mathrm{P} \approx 3 \mathrm{~mW}$ emitting a wavelength of $\lambda=650 \mathrm{~nm}$ was subjected to modulation. The laboratory stand was created using commercial Virtual Battlespace 3 system (VBS3), which has been supplemented by encoding device developed by the authors, Fig. 3, as well as laser beam decoding device. $V B S 3$ software was used to generate scenarios in which the $A R$ system affected the user. An example scene is shown in Fig. 4a. The generated image was transferred to the $7^{\text {th }}$ Sense Delta 2208 multimedia server. The server split the input image between the three projectors that displayed it at 1920 px x 1200 px. ProjectionDesign F35 AS3D WUXGA projectors were used in the study as well as a half cylindrical screen, Fig. $4 \mathrm{~b}$, measuring $12 \mathrm{~m} \times 3 \mathrm{~m}$. The user was about $3 \mathrm{~m}$ from the surface of the screen. During the test, the player was equipped with a Smith \& Wesson Springfield weapon model, supplemented with the coding system shown in Fig. 3a. The task of the player was to shoot in strictly defined situations, enforced by the $A R$ system. These shots were observed by the camera and then decoded in real time. 


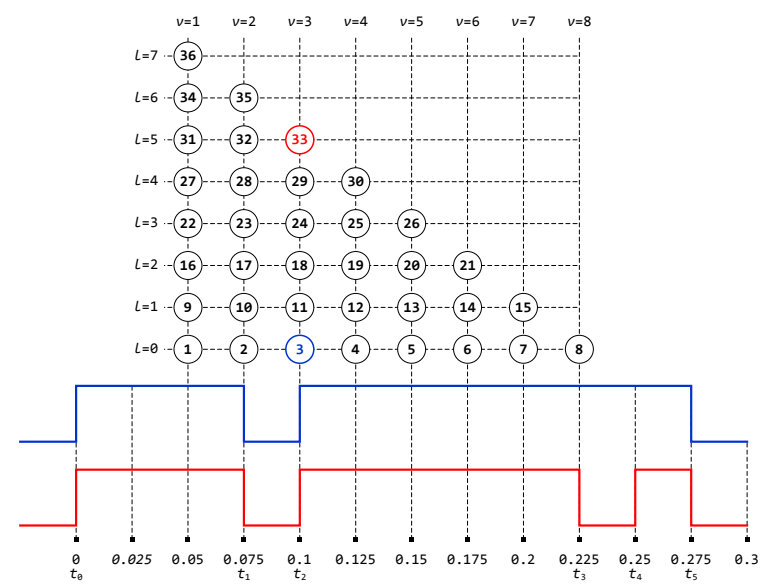

Fig. 5 Diagram of encoding symbols and code frame for symbol 33

\section{METHOD OF LASER BEAM CODING}

During developing the laser signal coding method, $N=36$ encoded symbols were assumed. The default state of the laser module is "off", which is manifested by the lack of a laser spot on the screen, and thus on the image. At the same time, it was assumed that displaying a single bit frame of code $T_{W}$, frame format $\left[D_{T}, D_{l}, \ldots, D_{L+1}, D_{P}, D_{E}\right]$, where $D_{T}-$ start bit, $D_{P}-$ stop bit, $D_{E}-$ frame end bit, $D_{k}-$ code bit for $1 \leq k \leq L+1$. Parameter $L$ is dependent on $N$ and is equal to $L=\operatorname{round}(\operatorname{sqrt}(2 N))$. In the layout of Fig. 2, the next frame bits of code is formed by terminal P1.2, which is responsible for turning on and off the laser module. The start bit and stop bit always have a value of one. The $D_{E}$ bit always assumes zero. This corresponds to the transition from the state of emission of subsequent frame bits to the deactivation of the laser module. The impact of the $T_{W}$ bit emission time on the effectiveness of decoding transmitted symbols has been verified in the studies of the coding system. The dependency of the required time of emitting the $T_{W}$ bit as a function of the frequency of images obtained from the camera was also determined. The principle of encoding a frame is presented in Fig. 5. Thirty six symbols, $N=36$, were arranged on eight levels $L=8$. At the level of index $l$ equaling zero, eight symbols were placed. Each higher level contains one symbol less. At the last level $l=L-1$ only one symbol is placed. For each symbol, for each level, a sequence number $v$ is assigned. The coded symbol can then be written in the form

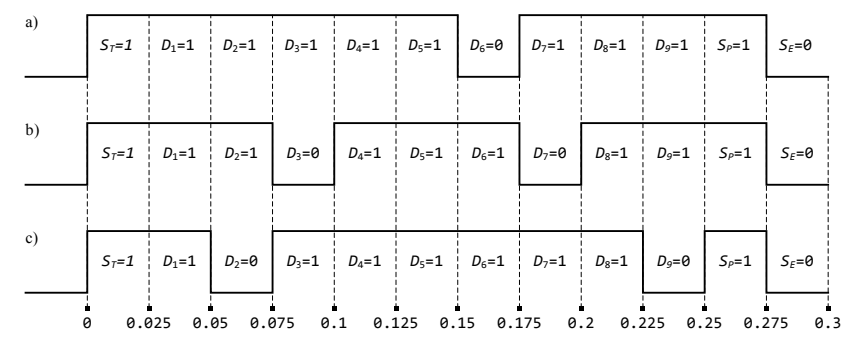

Fig. 6 Coding example: a) 6 symbols $(v=6 ; 1=0)$; b) 24 symbols $(\mathrm{v}=3,1=3) ; \mathrm{c}) 35$ symbols $(\mathrm{v}=2 ; 1=6)$ of $S=v+0.5 l(2 L+1-l)$, where $0 \leq l<L$. The pulse width depends on parameters $v$ and $l$, and the pause time between pulses does not change and equals $T_{W}$. An example of symbol encoding from level $l=5$ (first from the bottom) and a symbol from level $l=0$ (second from the bottom) is shown in Fig. 5. In this example, time $T_{W}$ was equal to $0.025 \mathrm{~s}$.

The encoding of parameter $v$ is done by assigning a value of one in the code frame for bits $D_{1}$ to $D_{v-1}$ and the value of zero for $D_{v}$. If the symbol is at level $l=0$ then the bits from $D_{v+1}$ to $D_{L+1}$ are assigned a value of one. Otherwise, bits from $D_{v+1}$ to $D_{v+l}$ are set to one and the $D_{v+l+1}$ bit value is set to zero, which corresponds to the coding of the $l$ parameter. The other code bits are assigned a value of one. In both cases, the stop bit always assumes a value of one and the frame ends the frame end bit of $D_{E}=0$. Giving a logic zero to the end of P1.2 of the microcontroller disables the laser module. An example of symbol encoding: 6, 24 and 35 is shown in Fig. 6. Taking into account the start and stop bits values (always equal to one), it can be noticed that the width of the first generated pulse is $v T_{W}$. For symbols from level $l=0$ the second pulse width is equal $(L+2-v) T_{W}$, and for the remaining levels $l T_{W}$. The third pulse is generated only for symbols from levels $0<l<L$. Its width is equal to $(L+1-v-l) T_{W}$.

\section{METHOD OF DECODING OF LASER BEAM}

Decoding the symbol is carried out on the basis of analysis of the sequence of images obtained from the camera. Firstly, the function of laser module activation $u(t)$ is determined. Decoding the symbol takes place using the extended finitestate machine.

\section{A. CALCUlation OF LASER ACTIVATION FUNCTION}

Due to the use of the banded optical filter, only the red $R$ component of the image is used for further analysis. The luminance component is analyzed when using a monochrome camera. It is represented in matrix form in calculations ( $R$ matrix). The value of the laser module activation function $u(t)$ is determined in discrete moments of the time $n T_{S}$, $n \in N^{+}$based on the values of the elements of the $R$ matrix. The pseudo code appropriate for the algorithm determining the values of $u(t)$ is presented in Fig. 7. Firstly, maximizing

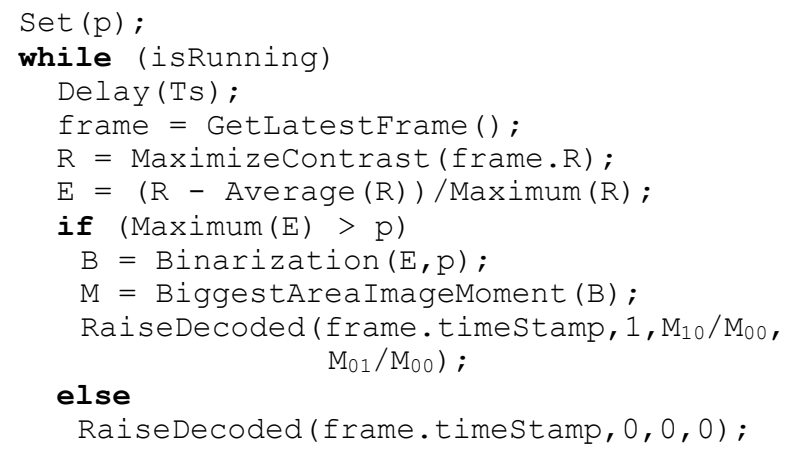

Fig. 7 Pseudo code to determine the value of the activation function 


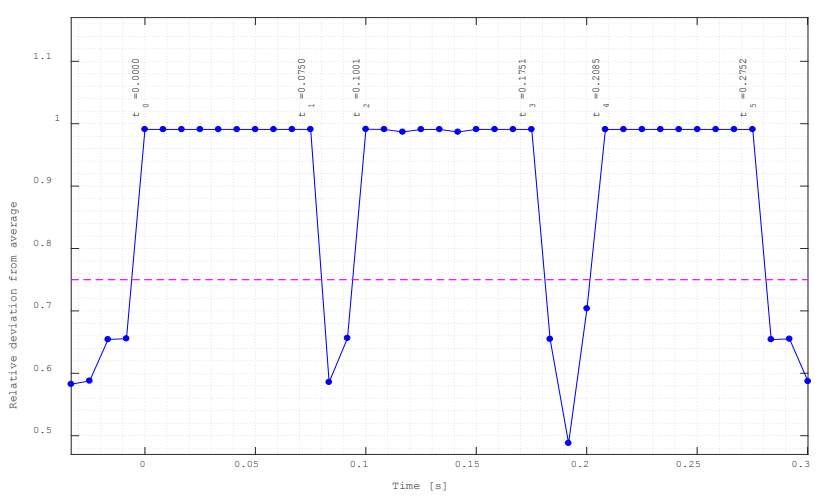

Fig. 8 Maximum value of the relative deviation from the mean in the function of time for $\mathrm{S}=24$ and $\mathrm{T}_{\mathrm{W}}=0.025 \mathrm{~s}$

the contrast of the color red is performed. Then, for matrix $R$, the matrix of relative deviations from the mean $R$ is determined, the values of which are determined from formula $e_{i j}=\left(r_{i j}-r_{a v g}\right) / r_{\max }$, where: $r_{i j}-$ is the element of matrix $R$ with coordinates $i, j$ obtained by the contrast maximization operation, $i, j-$ line and column numbers respectively of matrix $R, r_{a v g}$-average value of matrix $R$ (red color component after the contrast maximization operation), $r_{\max }-$ maximum value of the $R$ component of the image obtained after the image contrast maximization operation.

In the case of experimenting with a single shooter, at least one laser spot may be observed at a time in the image. This spot is determined based on the values of matrix $E$. When the maximum value of an element of matrix $E$ exceeds the value of the set threshold $p$, then the laser is considered to be enabled. At that time the activation function $u(t)$ assumes the value of one, or otherwise zero. The graph of the maximum relative deviation from the mean in the function of time recorded for symbol $S=24$ and the time $T_{W}=0.025 \mathrm{~s}$ is shown in Fig. 8. The detection of the laser spot forces the determination of matrix $B$ obtained during the binarization of the matrix $E$. Binarization is carried out in accordance with formula $b_{i j}=\left\{255\right.$, for $e_{i j}>p$; 0 , for $\left.e_{\mathrm{ij}} \leq p\right)$, where $p$ - the

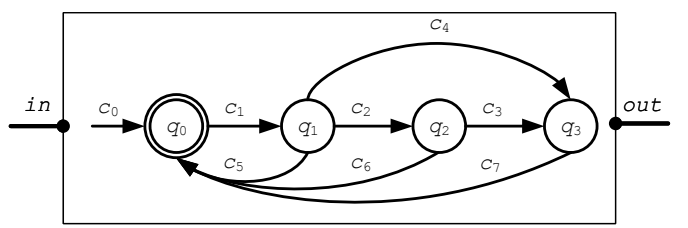

$C_{0}:\left(-,-,-, v:=0 \wedge I:=0 \wedge T_{E}:=0, \phi, q_{0}\right)$

$C_{1}:\left(q_{0}\right.$, in $\left.=[T, x, y], 0<f(T) \leq L, v:=f(T) \wedge T_{E}:=t i m e, \phi, q_{1}\right)$

$C_{2}:\left(q_{1}\right.$, in $\left.=[T, x, y], h(T) \leq L \wedge f\left(t i m e-T_{E}\right)=1, I:=f(T) \wedge T_{E}:=t i m e, \phi, q_{2}\right)$

$c_{3}:\left(q_{2}\right.$, in $=[T, x, y], h(T)=L+1 \wedge f\left(\right.$ time $\left.-T_{E}\right)=1, T_{E}:=$ time, out $\left.:=g(), q_{3}\right)$

$C_{4}:\left(q_{1}\right.$, in $=[T, x, y], h(T)=L+2 \wedge f\left(\right.$ time $\left.-T_{E}\right)=1, T_{E}:=$ time, out $\left.:=g(), q_{3}\right)$

$C_{5}:\left(q_{1},-, h\left(\right.\right.$ time $\left.\left.-T_{E}\right)+2>L, v:=0 \wedge T_{E}:=0, \phi, q_{0}\right)$

$C_{6}:\left(q_{2},-, h\left(\right.\right.$ time $\left.\left.-T_{E}\right)+1>L, v:=0 \wedge I:=0 \wedge T_{E}:=0, \phi, q_{0}\right)$

$C_{7}:\left(q_{3},-,-, v=0 \wedge l=0 \wedge T_{E}:=0, \phi, q_{0}\right)$

where:

$f(t)=\operatorname{round}\left(t / T_{N}\right)$

$h(t)=f(t)+v+1$

$g()=v+0.5 I(2 L+1-I)$

Fig. 9 Extended finite state machine given binarization threshold. The results of binarization are areas in matrix $B$ (spots), the largest of which determines the location of the laser pointer. It is for it image moments $\mathrm{M}_{00}$, $\mathrm{M}_{01}, \mathrm{M}_{10}$ and the center of gravity is calculated. In pseudocode, Fig. 7, the RaiseDecoded has been proposed to be used and transferred to the function of handling: the time stamp, the value of the activation function, and the coordinates of the center of gravity of the area identified with the laser pointer.

\section{B. EXTENDED FINITE STATE MACHINE}

Decoding the symbol is performed by the extended finitestate machine EFSM. At the EFSM input matrix $G$ with a structure of $[T, x, y]$ is given, where $T$ - pulse width, $x$ and $y$ - laser spot coordinates. In the accepted solution, matrix $G$ represents a single pulse extracted from the activation function of the laser module $u(t)$. According to the assumptions given in point IV, each symbol is encoded by several pulses (two or three pulses) depending on level $l$. Thus, based on state $q$ of the EFSM machine and the determined $G$ matrices, the symbols $S_{i} \in\left\{\phi, S_{1}, \ldots, S_{n}, \ldots, \mathrm{S}_{N}\right\}$ are indicated, where $\phi$ means "no symbol", $S_{1}, \ldots, \mathrm{S}_{N}$ represent decoded symbols, and $N$ specifies the allowed number of decoded symbols.

The extended finite-state machine was defined as 6-tuple $E F S M=\left(Q, q_{0}, V, I, O, C\right)$. The various symbols are: $Q-$ set of EFSM states; $q_{0}$ - initial state; $V-$ a set of EFSM variables; $I$-pulse matrix described in the form of $G ; O-$ a set of possible EFSM symbols; $A$-a set of allowed transitions between EFSM states. The diagram of states and elements of set $C$ are shown in Fig. 9. A set of $Q$ states of the EFSM machine is defined as $\left\{q_{0}, q_{1}, q_{2}, q_{3}\right\}$. State $q_{1}$ represents the position of the symbol $S$ defined within the coding level, and $q_{2}$ determines the level on which the symbol is located. The $q_{3}$ state is identified with turning off the laser module, and $q_{0}$ is the initial state of the EFSM. The set of variables $V$ is defined as $\left\{l, v, T_{E}\right.$, time, $\left.X, Y\right\}$, where the variables $l$ and $v$ indicate the parameters of symbol $S$, the variable $T_{E}$ defines the time of arrival at the input of matrix $G$, time indicates the elapsed time since appearing at the input of the matrix $G$, and $X$ and $Y$ denote the coordinates of the laser spot determined for the first pulse. Set $C$ is defined as $\left\{c_{k}:\left(q\right.\right.$, in, guard, $a$, out,$\left.\left.q^{\prime}\right)\right\}$ and means that if EFSM is in state $q$ and matrix $[T, x, y]$ appears at the in input, then the transition to the state of $q$ ' is possible provided that the logical expression guard is true. Only in this case will the searched for symbol $S$ be determined on the out output. The output of the machine from its initial state $q_{0}$ means putting forward hypothesis $H_{0}$ on symbol detection. In subsequent times, if the time limits are not met, the machine automatically returns to $q_{0}$, which is equivalent to rejecting hypothesis $H_{0}$. The assumption of hypothesis $H_{0}$ takes place only at the moment the machine goes into state $q_{3}$. Only then will symbol $S_{i}$ appear on the output, indicating its recognition. Determining the output symbol is based on 


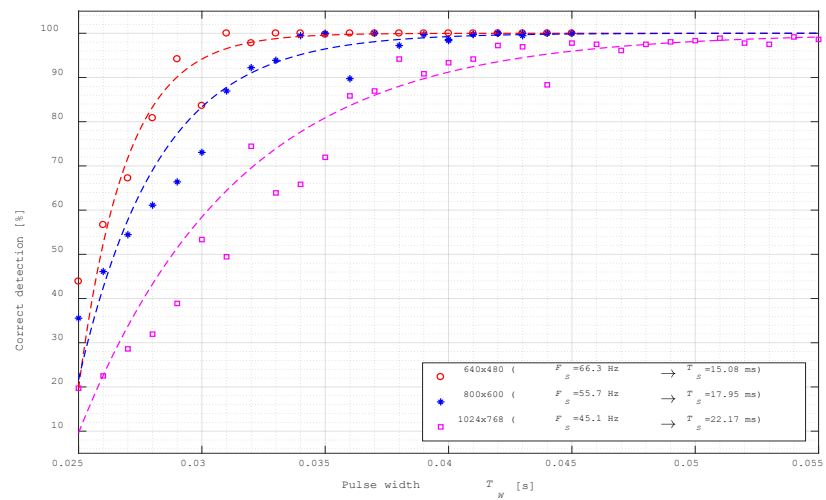

Fig. 10 Detection efficiency in the function of duration of bit $T_{W}$ and tested image resolutions

variables $v$ and $l$. In other cases, the EFSM output is given element $\phi$.

\section{RESULTS OF RESEARCH}

The experiment was carried out for a test set containing $N=36$ symbols, $S \in\left\{S_{n}: n=1, \ldots, N\right\}$. Each symbol was encoded with the developed technique (Section IV). In this way, codes were obtained, which individual bits control the laser module ("1" - ON, "0" - OFF). The test consisted of sending the test set $K=100$ times. Altogether 3,600 symbols were sent and decoded. The experiment was divided into two parts. The first part was the effect of time $T_{W}$ on the efficiency of detecting $S$. The study was conducted for three camera resolutions: 640 px x 480 px, 800 px x 600 px and 1024 px x 768 px. In each test, the duration of $T_{W}$ was changed and accepted values ranging from $0.025 \mathrm{~s}$ to $0.055 \mathrm{~s}$ with a step of $0.001 \mathrm{~s}$. For each trial, the detection efficiency of the transmitted symbol was determined as well as the average detection efficiency of symbols belonging to the test set, Fig. 10. For the resolution of 640 px x 480 px, images were acquired on an average of $0.015 \mathrm{~s}(F P S=66.66 \mathrm{~Hz})$. For $T_{W}$ of $0.030 \mathrm{~s}$, the detection efficiency was no worse than $90 \%$. Symbol detection efficiency at a level of $100 \%$ was achieved when images were acquired at $0.036 \mathrm{~s}-F P S$ equal

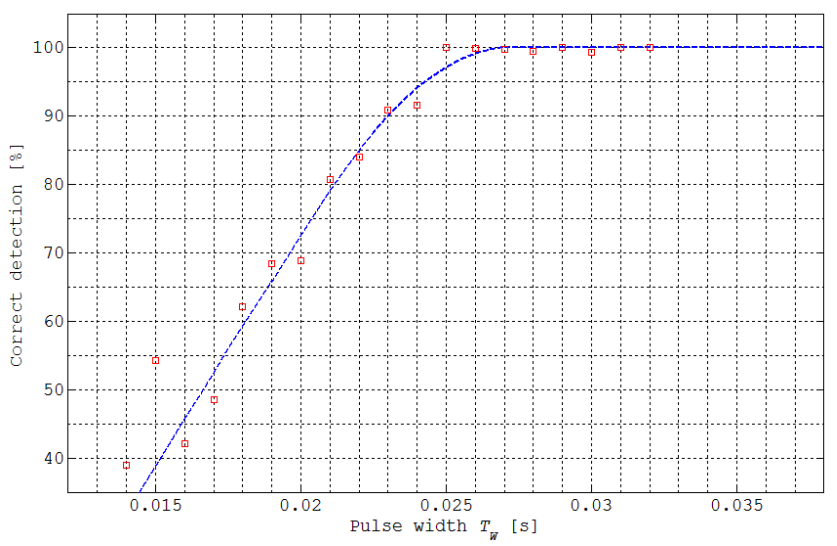

Fig. 11 Detection efficiency in the function of duration of bit $T_{W}$ for resolution $640 \mathrm{px}$ x $480 \mathrm{px}$ and $F P S=120 \mathrm{~Hz}$

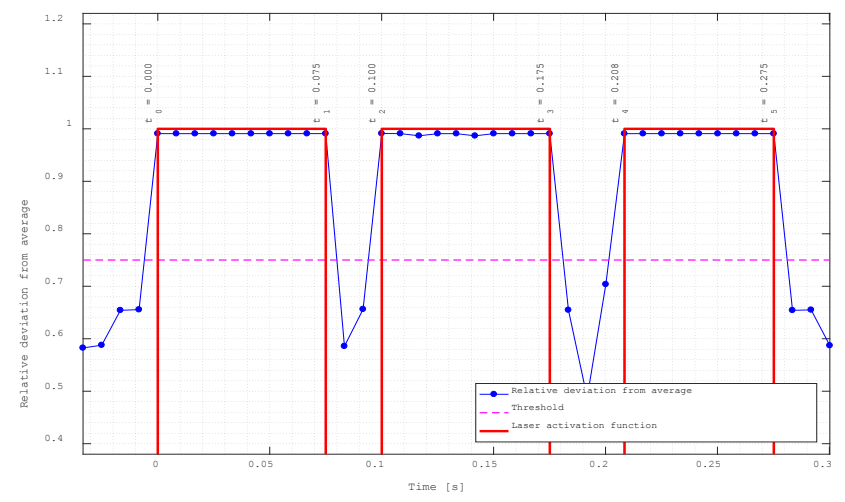

Fig. 12 Detection of symbol $\mathrm{S}_{24}, T_{W}=0.025 \mathrm{~s}$, image resolution 640 px x 480 px

27.77 Hz. Comparative dependencies were obtained for resolutions: 800 px x 600 px and 1024 px x 768 px.

The second part of the study used the OptiTrack 120 Slim miniature high speed camera for motion capture. The camera was equipped with a lens with a focal length of $F=16 \mathrm{~mm}$ and a bandpass filter of $\lambda_{0}=650 \mathrm{~nm}$ and $\Delta \lambda= \pm 10 \mathrm{~nm}$. The study was conducted for a resolution of $640 \mathrm{px}$ x $480 \mathrm{px}$ and $F P S=120 \mathrm{~Hz}$. The second part of the experiment was divided into two phases. Firstly, it was confirmed that $T_{W}$ has a similar effect on the effectiveness of the detecting symbols as was the case in the first part of the experiment. The study was performed by changing the duration of the $T_{W}$ bit from $0.014 \mathrm{~s}$ to $0.030 \mathrm{~s}$ with a step of $0.001 \mathrm{~s}$. In this case, for the time of $T_{w}$ greater than $0.025 \mathrm{~s}$, a decoding efficiency of the transmitted symbols was achieved of over $99 \%$. The obtained results are shown in Fig. 12. In the second phase, the effectiveness of symbol recognition for $T_{W}$ was $0.024 \mathrm{~s}$ and $0.025 \mathrm{~s}$. For $T_{W}=0.024 \mathrm{~s}$, the efficiency of symbol recognition was no worse than $91.67 \%$, Table I. Detailed results are shown in Table II. The lowest efficiency of $67 \%$ was achieved with symbol $S_{12}$. Efficiency of $100 \%$ was obtained for the symbols: $S_{1}, S_{2}, S_{3}, S_{7}, S_{8}, S_{9}, S_{14}, S_{15}, S_{16}$, $S_{21}, S_{24}, S_{34}, S_{36}$. No symbol has been misrecognized. In three hundred cases, the $q_{0}$ status was dropped, because no time limit was met, so no symbol was assigned. For $T_{W}$ greater than $0.025 \mathrm{~s}$, the detection efficiency was greater than $99 \%$. A detailed result of recognizing symbol $S_{24}$ is shown in Fig. 12. The laser module activation function consisted of three pulses. Parameters $v$ and $l$ of the symbol were determined based on this. Parameter $v$ was defined based on formula $v=\operatorname{round}\left(\left(t_{1}-t_{0}\right) / T_{W}\right)$, therefore $l=3$. Parameter $l$ was determined from the formula $l=\operatorname{round}\left(\left(t_{3}-t_{2}\right) / T_{W}\right)$,

TABLE I.

COLLECTIVE RESULTS OF DECECTING SYMBOLS FOR TW $=0.024 \mathrm{~S}$ AND AN IMAGE RESOLUTION OF 640 PX X 480 PX

\begin{tabular}{|l|c|}
\hline Number of symbols tested & 3600 \\
\hline Number of detections & 3300 \\
\hline Number of unrecognized symbols & 300 \\
\hline Efficiency & $91,67 \%$ \\
\hline
\end{tabular}


TABLE II.

DETAILED RESULTS OF DETECTING SELECTED SYMBOLS

\begin{tabular}{|c|c|c|c|}
\hline Symbol & $\begin{array}{c}\text { Number of } \\
\text { symbols in the } \\
\text { test set }\end{array}$ & $\begin{array}{c}\text { Number of } \\
\text { correct } \\
\text { detections }\end{array}$ & $\begin{array}{c}\text { Decoding } \\
\text { Efficiency } \\
\text { [\%] }\end{array}$ \\
\hline$S_{1}$ & 100 & 100 & 100 \\
\hline$S_{3}$ & 100 & 100 & 100 \\
\hline$S_{5}$ & 100 & 83 & 83 \\
\hline$S_{7}$ & 100 & 100 & 100 \\
\hline$S_{9}$ & 100 & 100 & 100 \\
\hline$S_{11}$ & 100 & 96 & 96 \\
\hline$S_{13}$ & 100 & 94 & 94 \\
\hline$S_{15}$ & 100 & 100 & 100 \\
\hline$S_{17}$ & 100 & 96 & 96 \\
\hline$S_{19}$ & 100 & 77 & 77 \\
\hline$S_{21}$ & 100 & 100 & 100 \\
\hline$S_{23}$ & 100 & 84 & 84 \\
\hline$S_{25}$ & 100 & 81 & 81 \\
\hline$S_{27}$ & 100 & 69 & 69 \\
\hline$S_{29}$ & 100 & 82 & 82 \\
\hline$S_{31}$ & 100 & 93 & 93 \\
\hline$S_{33}$ & 100 & 92 & 92 \\
\hline$S_{35}$ & 100 & 98 & 98 \\
\hline & & & \\
\hline
\end{tabular}

therefore $l=3$. The detected impulses fulfilled all time limits. On this basis, and based on the proposed coding scheme, Fig. 5, symbol $S_{24}$ was recognized.

\section{CONCLUSION}

The article presents methods of: encoding and decoding laser signals developed to control the system of augmented reality. Characteristics of the developed coding method are: constant code frame emission time, one or two intervals between pulses, tight time limits for pulse width and intervals between them. Constant time for frame emission equal to $(3+L) T_{W}$ to reduce the number of false detection of the transmitted symbol. Adopted strong limitations on pulse width reduce the number of type II errors, but force the use of cameras with a fixed image acquisition time. In turn, a small number of pauses between pulses allows for effective detection when symbols are emitted by multiple encoding devices.

It has been shown that using the proposed methods make it possible to distinguish between commands encoded in a laser beam with an efficiency of $99.9 \%$. In the case of using cameras with similar parameters to the units used, the duration of the $T_{W}$ bit should be no less than $3 T_{S}$, where $T_{S}$ represent the interval between the two acquired image frames.

The laser signal encoding algorithm can be successfully implemented in the embedded system. The implementation of the encoding algorithm required 57.1 bytes of data memory (byte and bit addressing memory) and 3,664 bytes of program memory.

\section{REFERENCES}

[1] D. R. Olsen, T. Nielsen, "Laser pointer interaction", in Proceedings of the SIGCHI conference on Human factors in computing systems, pp. 17 - 22, 2001. https://doi.org/10.1145/365024.365030.

[2] F. Vogt, J. Wong, S. Fels, D. Cavens, "Tracking Multiple Laser Pointers for Large Screen Interaction", Extended Abstracts of ACM UIST 2003, pp. 95 - 96, 2003.

[3] A. Soetedjo, E. Nurcahyo, "Developing of Low Cost Vision-Based Shooting Range Simulator", in IJCSNS International Journal of Computer Science and Network Security, vol. 11, no. 2, 2011.

[4] H. Ebrahimpour-Komleh, M. Tekiyehband, "Design of an interactive whiteboard system using computer vision techniques", Proceedings of 6th International Symposium on Mechatronics and its Applications 2009(ISMA '09), pp. 423 - 26, 2009.

[5] J.-t. Wang, C.-N. Shyi, T.-W. Hou, C. P. Fong, "Design and Implementation of Augmented Reality System Collaborating with QR Code", Computer Symposium (ICS), 2010 International, pp. $414-$ 418, 2010. https://doi.org/10.1109/COMPSYM.2010.5685477.

[6] H. Ukida, S. Kaji, Y. Tanimoto, H. Yamamoto, "Human Motion Capture System Using Color Markers and Silhouette", Instrumentation and Measurement Technology Conference, IMTC 2006, proceedings of the IEEE, pp. $151-156,2006$. https://doi.org/10.1109/IMTC.2006.328334.

[7] A. Smeragliuoloa, N. Hillc, L. Dislad, D. Putrino, "Validation of the Leap Motion Controller using markered motion capture technology", Journal of Biomechanics, vol. 49, 9, pp. 1742 - 1750, 2016. http://doi.org/10.1016/j.jbiomech.2016.04.006.

[8] K. Barczewska, A. Drozd, "Comparison of methods for hand gesture recognition based on Dynamic Time Warping algorithm" in Proceedings of the 2013 Federated Conference on Computer Science and Information Systems, pp. 207 - 210, 2013.

[9] J. Lebiedź, M. Szwoch, "Virtual Sightseeing in Immersive 3D Visualization Lab", in Proceedings of the 2016 Federated Conference on Computer Science and Information Systems, M. Ganzha, L. Maciaszek, M. Paprzycki (eds). ACSIS, vol. 8, pp. 1641-1645, 2016. http://dx.doi.org/10.15439/2016F227.

[10] T. Bothe, A. Gesierich, W. Li, C. Kopylow, N. Kopp, W. Juptner, "3D-Camera for Scene Capturing and Augmented Reality Applications", 3DTV Conference, pp. 1-4, 2007. https://doi.org/10.1109/3DTV.2007.4379469.

[11] T. Pałys, W. Żorski, "Enhanced movement tracking with Kinect supported by high-precision sensors", in Proceedings of the 2015 Federated Conference on Computer Science and Information Systems, M. Ganzha, L. Maciaszek, M. Paprzycki (eds). ACSIS, vol. 5, pp. 883 - 888, 2015. http://dx.doi.org/10.15439/2015F166.

[12] K. Murawski, A. Arciuch, T. Pustelny, "Studying the influence of object size on the range of distance measurement in the new Depth From Defocus method", in Proceedings of the 2016 Federated Conference on Computer Science and Information Systems, M. Ganzha, L. Maciaszek, M. Paprzycki (eds). ACSIS, vol. 8, pp. 817-822, 2016. doi: 10.15439/2016F136.

[13] N. W. Kim, H. Lee, "Developing of vision-based virtual combat simulator", in Proceedings of International Conference on IT Convergence and Security (ICITCS), Macao, China, pp. 1- 4, 2013.

[14] GKDesign Engineering: "RS-232 Laser Transceiver", Electronics Australia, pp. $56-61,1997$.

[15] K. Murawski, R. Różycki, P. Murawski, A. Matyja, M. Rekas, "An Infrared Sensor for Monitoring Meibomian Gland Dysfunction", in Acta Physica Polonica A, vol. 124, no 3, pp. 517 - 520, 2013. doi: 10.12693/APhysPolA.124.517.

[16] K. Murawski, "Measurement of membrane displacement with a motionless camera equipped with a fixed focus lens", Metrology and Measurement Systems, vol. 22, no. 1, pp. 69-78, 2015. doi.: 10.1515/mms-2015-0011

[17] K. Murawski, "New Vision Sensor to Measure and Monitor Gas Pressure", Acta Physica Polonica A, vol. 128, no 1, pp. 6- 9, 2015. doi: 10.12693/APhysPolA.128.6

[18] K. Murawski, "Measurement of membrane displacement using a motionless camera", Acta Physica Polonica A, vol. 128, no 1, pp. 10 - 14, 2015. doi: 10.12693/APhysPolA.128.10 